\title{
Predicting 30-Day All-Cause Readmission Risk for Subjects Admitted With Pneumonia at the Point of Care
}

\author{
Umur Hatipoğlu MD, Brian J Wells MD PhD, Kevin Chagin MSc, Dhruv Joshi MD, \\ Alex Milinovich, and Michael B Rothberg MD MPH
}

\begin{abstract}
BACKGROUND: The pneumonia 30-d readmission rate has been endorsed by the National Quality Forum as a quality metric. Hospital readmissions can potentially be lowered by improving inhospital care, transitions of care, and post-discharge disease management programs. The purpose of this study was to create an accurate prediction model for determining the risk of 30-d readmission at the point of discharge. METHODS: The model was created using a data set of 1,295 hospitalizations at the Cleveland Clinic Main Campus with pneumonia over 3 y. Candidate variables were limited to structured variables available in the electronic health record. The final model was compared with the Centers for Medicare and Medicaid Services (CMS) model among subjects $65 \mathrm{y}$ of age and older $(n=628)$ and was externally validated. RESULTS: Three hundred thirty subjects $(25 \%)$ were readmitted within $30 \mathrm{~d}$. The final model contained 13 variables and had a bias-corrected C statistic of 0.74 (95\% CI 0.71-0.77). Number of admissions in the prior 6 months, opioid prescription, serum albumin during the first $24 \mathrm{~h}$, international normalized ratio and blood urea nitrogen during the last $24 \mathrm{~h}$ were the predictor variables with the greatest weight in the model. In terms of discriminative performance, the Cleveland Clinic model outperformed the CMS model on the validation cohort (C statistic 0.69 vs $0.60, P=.042$ ). CONCLUSIONS: The proposed risk prediction model performed better than the CMS model. Accurate readmission risk prediction at the point of discharge is feasible and can potentially be used to focus post-acute care interventions in a high-risk group of patients. Key words: pneumonia readmission; prediction model; health-care utilization. [Respir Care 2018;63(1):43-49. (C) 2018 Daedalus Enterprises]
\end{abstract}

\section{Introduction}

An unplanned hospital readmission within $30 \mathrm{~d}$ of discharge after an acute hospitalization has been endorsed as an important quality indicator for public health reporting

Drs Hatipoğlu and Joshi are affiliated with the Respiratory Institute, Mr Chagin and Mr Milinovich are affiliated with Quantitative Health Sciences, and Dr Rothberg is affiliated with the Center for Value-Based Care Research, Medicine Institute, Cleveland Clinic, Cleveland, Ohio. Dr Wells is affiliated with the Clinical and Translational Science Institute, Wake Forest University School of Medicine, Winston-Salem, North Carolina.

The authors have disclosed no conflicts of interest.

Dr Hatipoğlu presented a version of this work at the Chest 2015 International Conference, held October 25-28, 2015, in Montréal, Canada.

Supplementary material related to this paper is available at http:// www.rcjournal.com. purposes. ${ }^{1}$ Previous studies indicate that many readmissions are associated with suboptimal transitions of care and may be preventable. ${ }^{2,3}$ The Medicare Payment Advisory Commission has estimated that readmissions cost $\$ 15$ billion annually. ${ }^{4}$ The Centers for Medicare and Medicaid Services (CMS) launched the Hospital Readmissions Reduction Program, which requires CMS to reduce payment to participating hospitals with readmission rates in excess of the national average. The Hospital Readmissions Reduction Program has focused on diseases with the highest expenditures and readmission rates and for which adherence to management guidelines improves outcomes. One target condition, pneu-

\footnotetext{
Correspondence: Umur Hatipoğlu MD, Respiratory Institute-Cleveland Clinic, Desk A.90, 9500 Euclid Avenue, Cleveland, OH 44195. E-mail: hatipou@ccf.org.
}

DOI: $10.4187 /$ respcare.05719 
monia, had a 30-d readmission rate of $15.7 \%$ in $2010^{5}$ and cost $\$ 10.6$ billion for Medicare in 2011. ${ }^{6}$

There are a number of ways hospitals might reduce readmissions. Improvements in in-patient care, optimizing transitions, and appropriate discharge disposition are all associated with reduced readmission rates. ${ }^{7,8}$ However, comprehensive post-acute discharge programs are costly to implement for all patients. Identifying patients at the highest risk for readmission would allow for more efficient allocation of limited resources at the time of discharge. Amarasingham et $\mathrm{al}^{9}$ leveraged an electronic health record-based risk stratification algorithm to allocate limited resources to successfully prevent readmissions among patients with congestive heart failure in real time. The claims-based model used by the CMS to calculate hospital specific, risk-standardized pneumonia readmission rates was created to compare hospitals, not to identify high-risk patients. ${ }^{10}$ The CMS model does not include important clinical details, such as vital signs, medications, laboratory results, and in-patient treatments, such as oxygen therapy. Other studies have found that clinical predictor variables add to the accurate prediction of readmission risk. ${ }^{11,12} \mathrm{Fi}$ nally, the CMS model was created using data from a wide variety of clinical institutions and has poor discrimination (C statistic 0.63 ). In a specific hospital, it may not perform as well as a model created using local data.

The purpose of this study was to create a practical, local model for predicting the risk of 30-d all-cause readmission following admission for pneumonia. The model was not intended to determine causal inference but rather to create the most accurate risk assessment based on structured variables available in the electronic health record and therefore ready for use at the point of care.

\section{Methods}

\section{Setting and Subjects}

The study was approved by the institutional review board of the Cleveland Clinic (approval 13-382). The study was performed on a retrospective cohort of 1,421 adult hospitalizations age $\geq 18$ y who were admitted to the Cleveland Clinic Main Campus, a tertiary care academic medical center with 1,400 beds. The cohort consisted of subjects admitted with a primary diagnosis of pneumonia, identified by the International Classification of Diseases, 9th Revision (ICD-9) codes utilized in the CMS readmission metric (480.XX, 481, 482.XX, 483.X, 485, 486, 487.0), between January 2010 and December $2012 .{ }^{10}$ Manual chart review of the entire population was conducted to ensure validity of the outcome data (ie, readmission status). Patients who did not have a documented clinical follow-up in the Cleveland Clinic Health System between $30 \mathrm{~d}$ and 6 months after discharge were excluded. The final model

\section{QUICK LOOK}

\section{Current knowledge}

Pneumonia readmissions represent a significant burden to health-care expenditure and patient morbidity. Assessing risk of readmission during in-patient care may allow focusing intensive interventions to a select group of individuals who may benefit the most while optimizing use of limited resources.

\section{What this paper contributes to our knowledge}

This work describes a pneumonia readmission risk prediction model based on data readily available in the electronic health record at the point of care. The model had good discriminative performance and predicted risk accurately along a broad range. The model enables calculation of readmission risk at the point of discharge, potentially allowing intensive interventions to focus on high-risk patients.

was then validated on subjects admitted with a diagnosis of pneumonia to the Cleveland Clinic in 2013 (validation cohort, $n=393$ ). Figure 1 summarizes the inclusion and exclusion criteria for the derivation and validation cohorts.

\section{Risk Factors for Readmission}

Candidate variables were determined based on literature review and included age, ${ }^{12}$ sex, ${ }^{11,13,14}$ race, ${ }^{15}$ vital signs, ${ }^{16}$ immunization status, ${ }^{17}$ medications, ${ }^{17}$ and comorbidities. ${ }^{18}$ Variables had to be discretely and reliably available within the electronic health record. The complete list of 134 variables considered as candidates for the model building are displayed in supplementary materials at http://www. rcjournal.com. Univariate analyses were used to screen the complete list of candidate variables. Variables were removed if not significantly associated with the outcome $(P \leq .05)$ or if they displayed an SE around the estimated coefficient $>0.3$. A complete logistic model was then fit using the remaining variables with a clustering penalization for the repeating measures within each subject. ${ }^{19}$ The clustering term was added to the final model to account for repeated admissions by the same subject. Variables that appeared to be causing multicollinearity (as assessed by variable inflation factor $\geq 2$ ) were removed.

For variables with repeated measures, such as vital signs and laboratory results, the model focused on the periods of admission and discharge. Specifically, only the minimum (lowest) and maximum (highest) measurements recorded within the first and last $24 \mathrm{~h}$ of the hospital stay were included. This approach is similar to that of the APACHE 

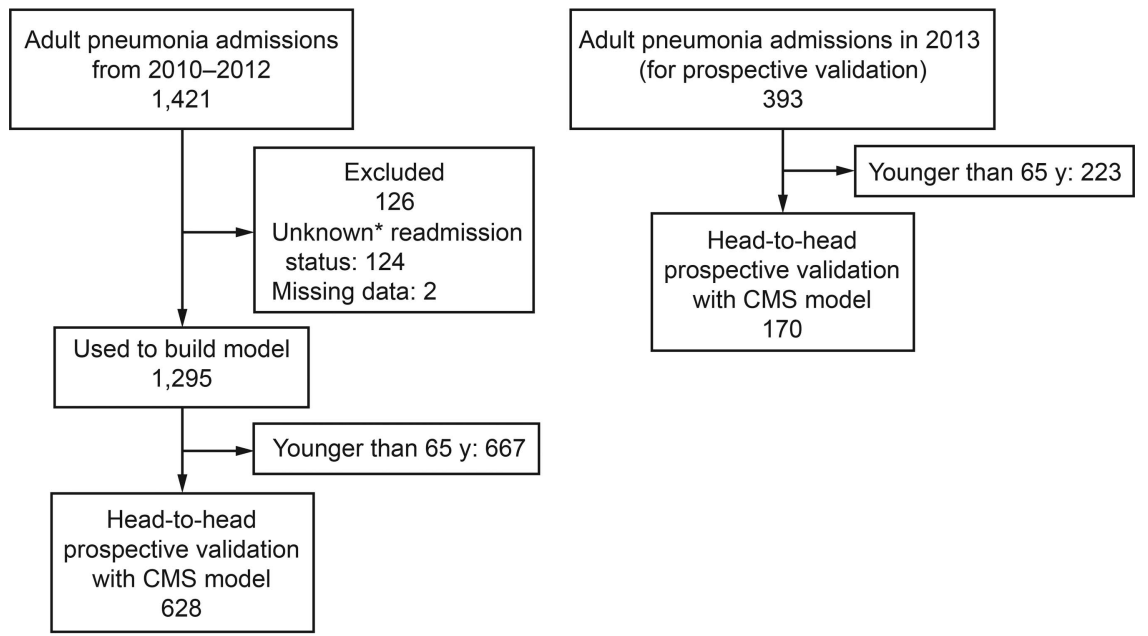

Fig. 1. Flow chart for the creation and validation of the Cleveland Clinic pneumonia model. * Twenty-seven of these patients died within $30 \mathrm{~d}$ of discharge according to the Ohio Death Index.

III calculator. ${ }^{20}$ Continuous variables were modeled using natural $\log$ transformations to create a linear relationship with the outcome. If a linear relationship could not be established, then the variable was modeled using restricted cubic splines with 3 knots.

Missing data were imputed using the MICE (Multiple Imputation by Chained Equations) package, version 2.22, for $\mathrm{R}$. The imputation process included all other predictor and outcome variables to build the regression equations used to replace the missing data. ${ }^{21}$ The imputation process was repeated 5 times with replacement. Harrell's ${ }^{22}$ model approximation process for variable selection was used to rank the variables in order of importance, per the model's $\mathrm{R}^{2}$. Discrimination was calculated using the concordance statistic, and random sampling with replacement was performed 1,000 times to obtain the optimized corrected concordance statistic at each ranking cutoff of the model selection process. The optimized corrected concordance statistic was plotted against model size, and the final model was chosen at the apex of this curve (ie, the final model included variables that maximize the discrimination, as measured by the concordance statistic).

The model was compared head-to-head with the Medicare administrative model. ${ }^{10}$ The Medicare prediction was calculated using a "cross-walk" file (available at https://www.cms. gov/Medicare/Health-Plans/MedicareAdvtgSpecRateStats/ Risk-Adjustors.html) that converts ICD-9 codes to the hierarchical conditional categories used in the Medicare calculation. The comparison with CMS was performed among the subset of subjects $65 \mathrm{y}$ of age and older during both the internal validation $(n=628)$ and the external validation $(n=170)$.

The models' discriminative performances were assessed by plotting receiver operating characteristic curves. In addition to discrimination, the accuracy of the final model was evaluated using a calibration curve. All statistical analyses were performed using R 2.15.2.

\section{Results}

Of the 1,421 hospitalizations, 2 were excluded because $>50 \%$ of the data points were missing, and 124 were excluded because their readmission status could not be determined from the electronic health record due to a lack of follow-up information. The investigators speculated that patient deaths occurring outside the hospital could partially explain the lack of follow-up data for these subjects. A search of the Ohio Death Index indicated that 27 of 124 subjects without follow-up electronic health record data had died within $30 \mathrm{~d}$ of discharge (22\%). Of the remaining 1,295 subjects, 299 were readmitted to a Cleveland Clinic hospital within $30 \mathrm{~d}$. The chart review identified 31 additional subjects who were admitted to other hospitals within $30 \mathrm{~d}$ (overall readmission rate $330 / 1,295=25 \%$ ). There were 1,191 unique subjects in the cohort, with 104 subjects experiencing 2 or more hospitalizations and 3 subjects experiencing 4 hospitalizations. Twenty-two of the 330 readmitted subjects $(6.6 \%)$ died during the readmission. All of the non-readmitted subjects were alive at the end of the 30-d period.

Univariate associations between risk factors and readmission appear in supplementary materials at http:// www.rcjournal.com. Readmitted subjects were older (66 y vs $62 \mathrm{y}, P<.001)$, had a longer stay $(7.2 \mathrm{~d}$ vs $5.5 \mathrm{~d}$, $P<.001$ ); were more likely to have cancer (all types combined), coronary heart disease, chronic heart failure, and chronic kidney disease $(P=.001, P<.001, P<.001$, and $P<.001$, respectively); and were more likely to receive prescriptions for warfarin or an opioid upon discharge (both $P<.001$ ). Among the laboratory values, 
Table 1. Final Model

\begin{tabular}{lcc}
\hline \hline \multicolumn{1}{c}{ Variables in the Cleveland Clinic Model } & Coefficient & $P$ \\
\hline Intercept & 12.3 & .03 \\
Age & 0.0143 & .02 \\
Cancer (yes) & 0.229 & .10 \\
CHD (yes) & 0.221 & .17 \\
Stroke (yes) & -0.192 & .29 \\
Time until first administered antibiotics (min)* & 0.140 & .09 \\
Opioids & 0.375 & .03 \\
Maximum temperature within the first $24 \mathrm{~h}$ & -0.104 & .03 \\
Maximum BUN within the last $24 \mathrm{~h}(\mathrm{mg} / \mathrm{dL})$ & 0.308 & .007 \\
Minimum sodium within the last $24 \mathrm{~h}(\mathrm{mmol} / \mathrm{L})$ & -0.0358 & .08 \\
Minimum hemoglobin within the last $24 \mathrm{~h}(\mathrm{~g} / \mathrm{dL})$ & -0.130 & .004 \\
Maximum albumin within the first $24 \mathrm{~h}(\mathrm{~g} / \mathrm{dL})$ & -0.342 & .009 \\
Minimum INR within the last $24 \mathrm{~h}$ & 0.320 & .01 \\
Total number of prior admissions within $6 \mathrm{mo}$ & 0.689 & $<.001$ \\
$\quad$ of admission & & \\
Total number of prior admissions within $6 \mathrm{mo}$ & -1.03 & $<.001$ \\
$\quad$ of admission $\dagger$ & & \\
& & \\
* Variables transformed using natural log. & & \\
$\dagger$ Variables fitted with a restricted cubic spline. & & \\
CHD = coronary heart disease \\
BUN = blood urea nitrogen \\
INR = international normalized ratio \\
\hline
\end{tabular}

lower values of hemoglobin and albumin within the first and last $24 \mathrm{~h}$ of hospitalization were also associated with higher readmission rates. Prior health-care utilization indices, such as prior hospital admissions, were also higher in those subjects with 30-d readmissions (2.96 vs 1.46 prior admissions over the past year, $P<.001)$. The final model contained 13 variables (Table 1 ) and had an internally validated, bias-corrected C statistic of 0.74 (95\% CI 0.71-0.77). In the external validation cohort from 2,013 subjects $(n=393)$, the C statistic was 0.71 .

The calibration curve suggests good calibration (Fig. 2). The model tended to overestimate risks in the highest risk subjects, and half of the risk probabilities fell between 0.08 and 0.39 . In the external validation cohort, the Cleveland Clinic model displayed better discrimination among patients $65 \mathrm{y}$ of age and older compared with the CMS model (C statistic 0.69 vs $0.60, P=.042$ ) (Fig. 3). Although both models had good calibration, the Cleveland Clinic model had a wider range of predicted probability $(0-0.8)$ than the Medicare administrative model (0.1-0.5). This allows for better discrimination of the higher- and lower-risk subjects. The upper 25 th percentile of the predicted risk according to the Cleveland Clinic Model is $>0.4$, with a sensitivity and specificity of 48 and $83 \%$, and the upper 25th percentile of the CMS predicted risk is $>0.29$, with a sensitivity and specificity of 31 and $81 \%$.

\section{Discussion}

In this retrospective study, we derived and validated a model for predicting 30-d readmission for patients hospi-

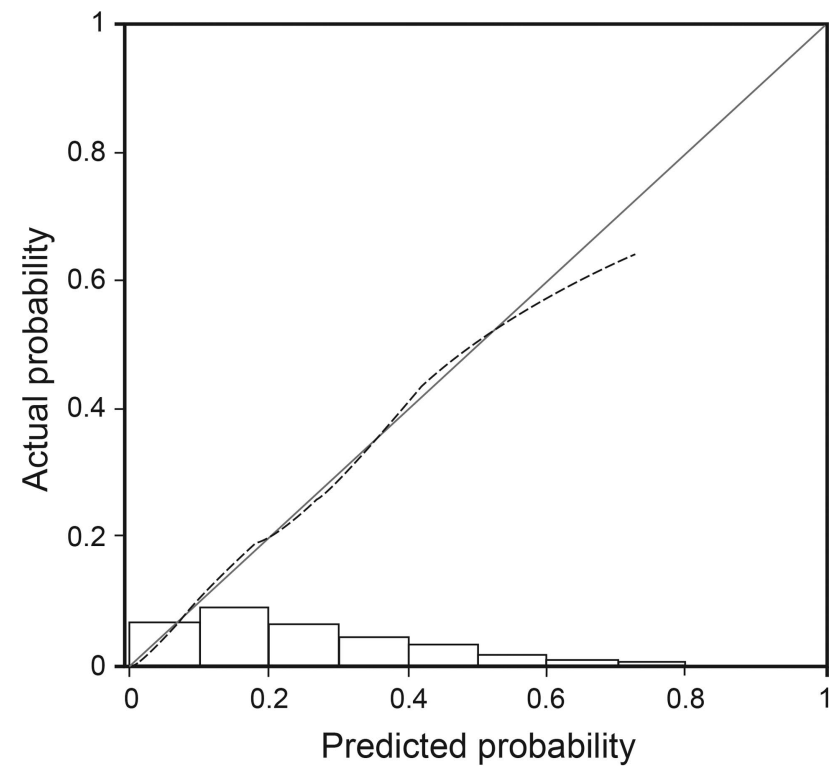

Fig. 2. Calibration curve for the final Cleveland Clinic pneumonia model. The $45^{\circ}$ line indicates perfect calibration, and any deviation from the ideal line indicates a difference between the predicted probability and the actual probability. Histograms indicate the number of subjects in each risk stratum. C statistic $=0.74(95 \% \mathrm{Cl}$ 0.71-0.77).

talized with pneumonia. Our model contained 13 variables, all of which are readily available in the electronic health record, and was better at identifying high-risk subjects than the CMS model. One reason for the model's superior performance was the inclusion of clinical variables that are not available in administrative data. In fact, 2 of the 3 most predictive variables (opioid prescription and serum albumin during the first $24 \mathrm{~h}$ ) were not administrative in nature. Overall, 9 of the 13 variables that contributed to the final model are not included in the CMS model. One reason that CMS uses administrative data for its model is the ease of collection. However, for real-time decision-making, discrete data points that can be extracted from the electronic health record may be easier to use, in addition to being more accurate. For the purpose of discriminating individual patients at high risk for readmission at the point of care, inclusion of clinical data improved model performance.

Readmissions following an index pneumonia admission are common, with approximately $16 \%$ of patients readmitted within $30 \mathrm{~d}^{5}$ The rate of readmission in the current study $(25 \%)$ is substantially higher than the national average, probably due in part to the complexity of patients seen at tertiary care centers like the Cleveland Clinic. Other factors that account for the difference may include a patient population with greater sociodemographic risk factors and a higher proportion of subjects susceptible to pneumonia complications, such as transplant recipients and cancer patients. Regardless, the differences between hos- 


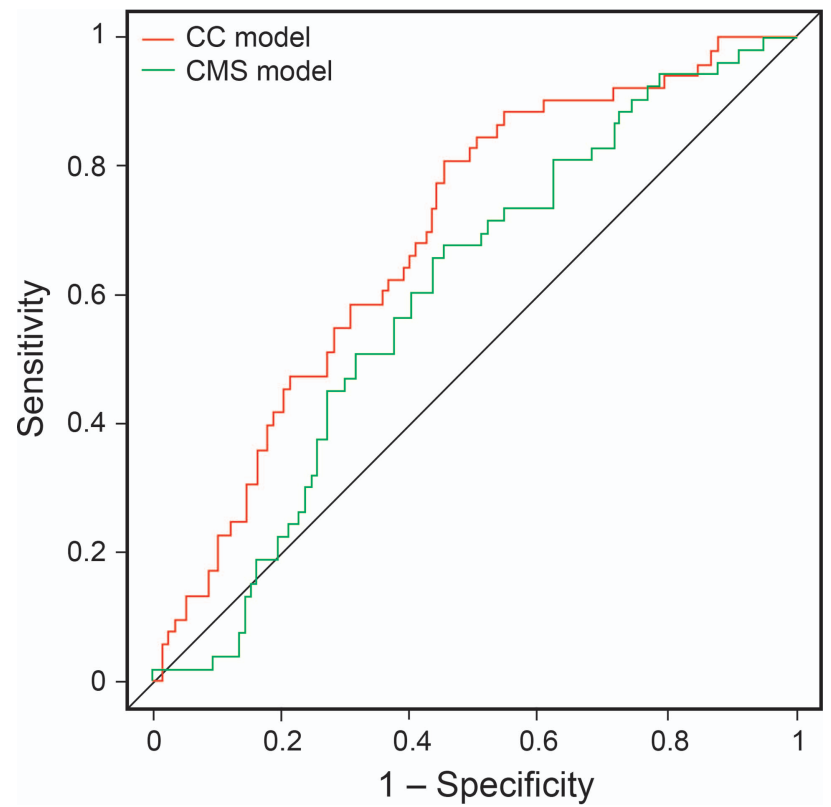

Fig. 3. Receiver operating characteristic curves for measuring the sensitivity and specificity of the Cleveland Clinic (CC) model and the Centers for Medicare and Medicaid Services (CMS) model's ability to predict the risk of readmission in a subset of subjects age $\geq 65$ y admitted with pneumonia in the 2013 validation set. $P=.042$. Area under the curve $=0.69$ for the CC model and 0.60 for the CMS model.

pitals highlights the potential benefits of locally created risk models.

Various studies have assessed factors associated with readmission. Halm et al ${ }^{16}$ reported that having one or more clinical criteria for instability (abnormal heart rate, breathing frequency, systolic blood pressure, temperature, low oxygen saturation, inability to swallow, and neurological impairment) during the $24 \mathrm{~h}$ before discharge was associated with a significantly higher risk of death and readmission. Some studies have identified modifiable risk factors, such as administration of appropriate antibiotics, ${ }^{7}$ vaccination status, ${ }^{17}$ and appropriate discharge planning. ${ }^{8}$ Other studies have noted the importance of non-modifiable factors, such as race ${ }^{15}$ and socioeconomic status. ${ }^{23,24}$ In building a high performance predictive model for pneumonia readmissions, one should be inclusive of all possible variables that may contribute to the risk of readmission. The model for pneumonia readmission developed in this paper considered a wide range of variables, including vital signs, laboratory findings, comorbid conditions, medications, and demographic data located within the electronic health record. The resulting model had a moderate to high concordance statistic and good calibration.

In a systematic review of clinical prediction models for various disease states, Kansagara et al ${ }^{25}$ identified 26 unique models for various diseases that used hospital readmission as the primary outcome. Nine used administrative data from multiple centers and displayed poor discriminative ability, raising questions about the validity of applying predicted risk models based on heterogeneous data for estimation of risk in individual hospitals. To that end, compared with the predictive model used to calculate the risk of readmission following hospitalization for pneumonia among Medicare beneficiaries, ${ }^{10}$ the Cleveland Clinic model performed better in discriminating between subjects with low and high risk for readmission. It is important to note that the model was built on the entire population of pneumonia subjects and was not limited to the elderly.

In addition to the CMS model, there are 7 other pneumonia readmission prediction models in the literature. ${ }^{10-12,26-29}$ These prediction models and publications that report on the CMS model ${ }^{10,30}$ were recently reviewed. ${ }^{31}$ In general, predictive models were critiqued for not including severity-of-illness measures, data on in-patient course, and stability on discharge. ${ }^{31}$ Moreover, the models were not compared directly with each other, so it is impossible to know how they would perform in other institutions. Our model outperformed the CMS model, highlighting the importance of a locally derived model for the purposes of identifying high-risk patients. In comparison with the Hartford Hospital model developed by Mather et al, ${ }^{11}$ our model included all pneumonia hospitalizations rather than only CMS beneficiaries. Recently, Makam et al ${ }^{29}$ published a pneumonia-specific readmission risk prediction model utilizing electronic health record data from the full hospital stay. Their model displayed good discrimination and predicted a broad range of risk similar to our model, but there are some notable differences in the choice of predictor variables. Specifically, Makam et al ${ }^{29}$ included median income of subjects as a predictor variable while excluding medications. Although socioeconomic status has been shown to be associated with readmission risk,,$^{23,24}$ inclusion of income may hinder point-of-care application, since this information may not be available through the electronic health record. In contrast, medications are readily available in most electronic health record systems and may provide important clues about severity of illness and potential adverse events.

Although interventions to specifically prevent pneumonia readmissions are scarce, ${ }^{32-34}$ focusing effective interventions to patients at high risk for readmission is a promising approach to reduce readmissions. ${ }^{9}$ Wasfy et al ${ }^{35}$ recently reported an accelerated reduction in readmission rates for pneumonia discharges after the passage of the Patient Protection and Affordable Care Act. This realworld report confirms findings from randomized control trials and instills further confidence in the efficacy of interventions to reduce readmissions. We propose that the next step is focusing resource-intensive interventions to high-risk patients by leveraging accurate risk prediction models. Interventions aimed at preventing readmissions 
may be improved if the reasons for readmissions are considered in the design of the intervention. An analysis of $>200,000$ readmissions within $30 \mathrm{~d}$ of an index hospitalization for pneumonia using Medicare claims also found a broad range of diagnoses, including pneumonia, heart failure, COPD, septicemia, renal disorders, cardiorespiratory failure, arrhythmias/conduction disorders, Clostridium difficile infections, urinary tract infections, and gastrointestinal bleeding. ${ }^{36}$ Given the significant heterogeneity in the reasons for readmission, interventions to prevent readmissions should include diverse elements to address the numerous readmission reasons.

Although causality should not be concluded from risk prediction paradigms, we note that opioid prescription on discharge was identified as a predictor variable for 30-d readmission in our cohort. Opioid-related adverse events and overdose are prevalent and pose a serious population health problem. ${ }^{37,38}$ In other settings, such as major abdominal surgery ${ }^{39}$ and liver transplantation, ${ }^{40}$ opioid prescription has been associated with increased $30-\mathrm{d}$ readmissions. To our knowledge, this is the first report of an association between opioid prescription on discharge and increased hospital readmission in the setting of an index pneumonia admission. Inclusion of opioid prescription as a predictor variable may improve performance among existing readmission prediction models for pneumonia.

Our study has limitations. First, we excluded patients whose readmission status could not be ascertained because they had no follow-up at the Cleveland Clinic after hospital discharge. This subgroup represented $<10 \%$ of the study population and was unlikely to affect overall model performance significantly. Additional sensitivity analysis revealed a similar predicted readmission risk for the cohort of subjects whose outcome was unknown compared with the subjects whose outcome was available to construct our model (data not shown). Second, we recognize that, although the model performed well in a validation cohort, continuous training may be necessary to maintain acceptable discriminative accuracy. ${ }^{27}$ Third, this model lacks validation beyond our hospital and may not be applicable to all settings. The users of the model can choose a probability threshold a priori, depending on desired sensitivity and specificity in predicting readmissions (ie, a higher probability threshold would identify fewer patients, but each would have a higher probability of readmission for more efficient use of resources). Finally, it should be noted that the model was created in adult subjects $\geq 18$ y of age, whereas the head-to-head validation with the CMS model was restricted to subjects $\geq 65 \mathrm{y}$ of age. Despite the differences in the composition of these cohorts in terms of age, the model still performed well.

\section{Conclusions}

We report a well-calibrated risk prediction model for pneumonia readmissions to the hospital with good discriminative capability, which could potentially be available at the point of discharge. The model was built on all hospitalized pneumonia subjects, not exclusively seniors. By utilizing a clustering term, we included all hospitalizations, including repeat hospitalizations of the same subjects. Discriminative performance was superior to the CMS risk prediction model among seniors. The model includes variables that are accessible and available in real time in the electronic health record. Therefore, the risk of readmission can be calculated at the point of discharge. We foresee such a calculator incorporated into the electronic health record and the calculated risk made available to care managers. The information could be used to focus post-acute care interventions in a high-risk group of patients. Further, the risk threshold could be adjusted to efficiently allocate available resources.

\section{REFERENCES}

1. NQF endorses all-cause unplanned readmissions measures. https:// www.qualityforum.org/News_And_Resources/Press_Releases/2012/ NQF_Endorses_All-Cause_Unplanned_Readmissions_Measures.aspx. Accessed June 21, 2017.

2. Oddone EZ, Weinberger M, Horner M, Mengel C, Goldstein F, Ginier P, et al. Classifying general medicine readmissions: are they preventable? Veterans Affairs cooperative studies in health services group on primary care and hospital readmissions. J Gen Intern Med 1996;11(10):597-607.

3. Halfon P, Eggli Y, Prêtre-Rohrbach I, Meylan D, Marazzi A, Burnand B. Validation of the potentially avoidable hospital readmission rate as a routine indicator of the quality of hospital care. Med Care 2006;44(11):972-981.

4. Report to the Congress: promoting greater efficiency in Medicare, June 2007, Chapter 5. http://www.medpac.gov/docs/default-source/ reports/Jun07_EntireReport.pdf?sfvrsn=0. Accessed June 21, 2017.

5. Readmissions to U.S. hospitals by diagnosis, 2010. https://www. hcup-us.ahrq.gov/reports/statbriefs/sb153.pdf. Accessed June 21, 2017.

6. National inpatient hospital costs: the most expensive conditions by payer, 2011. https://www.hcup-us.ahrq.gov/reports/statbriefs/ sb160.pdf. Accessed June 21, 2017.

7. Dean NC, Bateman KA, Donnelly SM, Silver MP, Snow GL, Hale D. Improved clinical outcomes with utilization of a communityacquired pneumonia guideline. Chest 2006;130(3):794-799.

8. Jack BW, Chetty VK, Anthony D, Greenwald JL, Sanchez GM, Johnson AE, et al. A reengineered hospital discharge program to decrease rehospitalization: A randomized trial. Ann Intern Med 2009; 150(3):178-187.

9. Amarasingham R, Patel PC, Toto K, Nelson LL, Swanson TS, Moore $\mathrm{BJ}$, et al. Allocating scarce resources in real-time to reduce heart failure readmissions: a prospective, controlled study. BMJ Qual Saf 2013 Dec;22(12):998-1005.

10. Lindenauer PK, Normand SL, Drye EE, Lin Z, Goodrich K, Desai $\mathrm{MM}$, et al. Development, validation, and results of a measure of 30-day readmission following hospitalization for pneumonia. J Hosp Med 2011;6(3):142-150. 


\section{Risk For 30-D ReAdmission in Subjects With Pneumonia}

11. Mather JF, Fortunato GJ, Ash JL, Davis MJ, Kumar A. Prediction of pneumonia 30-day readmissions: a single-center attempt to increase model performance. Respir Care 2014;59(2):199-208.

12. Capelastegui A, España Yandiola PP, Quintana JM, Bilbao A, Diez $\mathrm{R}$, Pascual S, et al. Predictors of short-term rehospitalization following discharge of patients hospitalized with community-acquired pneumonia. Chest 2009;136(4):1079-1085.

13. Neupane B, Walter SD, Krueger P, Marrie T, Loeb M. Predictors of in-hospital mortality and re-hospitalization in older adults with community-acquired pneumonia: a prospective cohort study. BMC Geriatr 2010;10:22.

14. Bohannon RW, Maljanian RD. Hospital readmissions of elderly patients hospitalized with pneumonia. Conn Med 2003;67(10):599603.

15. Joynt KE, Orav EJ, Jha AK. Thirty-day readmission rates for Medicare beneficiaries by race and site of care. JAMA 2011;305(7):675681.

16. Halm EA, Fine MJ, Kapoor WN, Singer DE, Marrie TJ, Siu AL. Instability on hospital discharge and the risk of adverse outcomes in patients with pneumonia. Arch Intern Med 2002;162(11):1278-1284.

17. El Solh AA, Brewer T, Okada M, Bashir O, Gough M. Indicators of recurrent hospitalization for pneumonia in the elderly. $\mathrm{J}$ Am Geriatr Soc 2004;52(12):2010-2015.

18. Fine MJ, Auble TE, Yealy DM, Hanusa BH, Weissfeld LA, Singer $\mathrm{DE}$, et al. A prediction rule to identify low-risk patients with community-acquired pneumonia. N Engl J Med 1997;336(4):243-250.

19. Feng Z, McLerran D, Grizzle J. A comparison of statistical methods for clustered data analysis with Gaussian error. Stat Med 1996;15(16): 1793-1806.

20. Knaus WA, Wagner DP, Draper EA, Zimmerman JE, Bergner M, Bastos PG, et al. The APACHE III prognostic system: risk prediction of hospital mortality for critically ill hospitalized adults. Chest 1991; 100(6):1619-1636.

21. Van Buuren S, Groothuis-Oudshoorn K. MICE: multivariate imputation by chained equations in R. J Stat Softw 2011;45(3):1-67.

22. Harrell FE Jr. Describing, resampling, validating and simplifying the model. In: Regression modeling strategies: with applications to linear models, logistic regression, and survival analysis, 2nd edition. New York: Springer; 2015:103-124.

23. Philbin EF, Dec GW, Jenkins PL, DiSalvo TG. Socioeconomic status as an independent risk factor for hospital readmission for heart failure. Am J Cardiol 2001;87(12):1367-1371.

24. Weissman JS, Stern RS, Epstein AM. The impact of patient socioeconomic status and other social factors on readmission: a prospective study in four Massachusetts hospitals. Inquiry 1994;31(2):163172.

25. Kansagara D, Englander H, Salanitro A, Kagen D, Theobald C, Freeman M, Kripalani S. Risk prediction models for hospital readmission: a systematic review. JAMA 2011;306(15):1688-1698.

26. Tang VL, Halm EA, Fine MJ, Johnson CS, Anzueto A, Mortensen EM. Predictors of rehospitalization after admission for pneumonia in the Veterans Affairs healthcare system. J Hosp Med 2014;9(6):379383.

27. Hebert C, Shivade C, Foraker R, Wasserman J, Roth C, Mekhjian H, et al. Diagnosis-specific readmission risk prediction using electronic health data: a retrospective cohort study. BMC Med Inform Decis Mak 2014;14:65.

28. Nagasako EM, Reidhead M, Waterman B, Dunagan WC. Adding socioeconomic data to hospital readmissions calculations may produce more useful results. Health Aff 2014;33(5):786-791.

29. Makam AN, Nguyen OK, Clark C, Zhang S, Xie B, Weinreich M, et al. Predicting 30-day pneumonia readmissions using electronic health record data. J Hosp Med 2017;12(4):209-216.

30. O'Brien WJ, Chen Q, Mull HJ, Shwartz M, Borzecki AM, Hanchate A, Rosen AK. What is the value of adding Medicare data in estimating VA hospital readmission rates? Health Serv Res 2015;50(1): 40-57.

31. Weinreich M, Nguyen OK, Wang D, Mayo H, Mortensen EM, Halm EA, Makam AN. Predicting the risk of readmission in pneumonia: a systematic review of model performance. Ann Am Thorac Soc 2016; 13(9):1607-1614.

32. Adamuz J, Viasus D, Simonetti A, Jiménez-Martínez E, Molero L, González-Samartino M, et al. Impact of an educational program to reduce healthcare resources in community-acquired pneumonia: the EDUCAP Randomized Controlled Trial. PLoS One 2015;10(10): e0140202.

33. McLeod-Sordjan R, Krajewski B, Jean-Baptiste P, Barone J, Worral P. Effectiveness of patient-caregiver dyad discharge interventions on hospital readmissions of elderly patients with community acquired pneumonia: a systematic review. JBI Libr Syst Rev 2011;9(14):437463.

34. Borzecki AM, Chen Q, Restuccia J, Mull HJ, Shwartz M, Gupta K, et al. Do pneumonia readmissions flagged as potentially preventable by the 3M PPR software have more process of care problems? A cross-sectional observational study. BMJ Qual Saf 2015;24(12):753763.

35. Wasfy JH, Zigler CM, Choirat C, Wang Y, Dominici F, Yeh RW. Readmission rates after passage of the Hospital Readmissions Reduction Program: a pre-post analysis. Ann Intern Med 2017;166(5): 324-331.

36. Dharmarajan K, Hsieh AF, Lin Z, Bueno H, Ross JS, Horwitz LI, et al. Diagnoses and timing of 30-day readmissions after hospitalization for heart failure, acute myocardial infarction, or pneumonia. JAMA 2013;309(4):355-363.

37. Macintyre PE, Huxtable CA, Flint SL, Dobbin MD. Costs and consequences: a review of discharge opioid prescribing for ongoing management of acute pain. Anaesth Intensive Care 2014;42(5):558574

38. Martins SS, Sampson L, Cerdá M, Galea S. Worldwide prevalence and trends in unintentional drug overdose: a systematic review of the literature. Am J Public Health 2015;105(11):2373.

39. Cron DC, Englesbe MJ, Bolton CJ, Joseph MT, Carrier KL, Moser $\mathrm{SE}$, et al. Preoperative opioid use is independently associated with increased costs and worse outcomes after major abdominal surgery. Ann Surg 2017;265(4):695-701.

40. Rogal S, Mankaney G, Udawatta V, Good CB, Chinman M, Zickmund $\mathrm{S}$, et al. Association between opioid use and readmission following liver transplantation. Clin Transplant 2016;30(10):1222-1229. 